\title{
Slicing into stroke therapeutics
}

Neuroprotection remains a key goal for stroke therapy, yet the lack of clinically effective neuroprotective drugs suggests that innovative strategies for the selection of target pathways and lead compounds are needed. A new approach, published by Lo and colleagues in PNAS, could help to achieve this and has already revealed an unexpected new target for ischaemic stroke therapy.

Much of the damage caused by stroke results from interruption of the blood supply, and consequently glucose and oxygen delivery, in affected brain areas. In this study, potentially neuroprotective compounds were tested in rat brain slices subjected to oxygen-glucose deprivation (OGD). To monitor neuronal damage, the slices were transfected with the gene for yellow fluorescent protein, which labelled a subset of cortical neurons that acted as 'sentinels' in providing a measure of neuronal survival. This slice-based approach provides a cellular environment similar to that found in vivo, yet allows relatively large numbers of compounds to be tested in the context of drug discovery programmes.

A collection of 1,200 compounds, selected for their drug-like properties, was examined using this method. This approach allowed a broad range of target pathways to be investigated, including many that have not been associated with neuroprotection, increasing the chances of identifying a new target. Importantly, the drugs were tested for neuroprotective effects when added at delayed time points after OGD onset, reflecting the probable time-frame for their use in stroke patients. The most effective compound was the cardiac glycoside neriifolin, which provided neuroprotection when tested more than 6 hours after OGD. The neuroprotective action of neriifolin was subsequently confirmed in a second brain-slice model and in two in vivo models of ischaemia. Significant but less potent neuroprotection by other cardiac glycosides was also demonstrated.

Cardiac glycosides, which are used clinically to treat cardiac dysfunctions, block $\mathrm{Na}^{+} / \mathrm{K}^{+}$-ATPase, an enzymatic pump that maintains ion concentration gradients across cell membranes. The demonstration of neuroprotection by cardiac glycosides was unexpected because many of these compounds can be toxic in other situations. However, the authors propose that the drugs might reduce cellular metabolic demand, or counteract a drop in calcium levels caused by ischaemia.

This study suggests that $\mathrm{Na}^{+} / \mathrm{K}^{+}$ATPase might be a promising target for neuroprotection, although further work will be needed to determine whether the delayed efficacy of neriifolin can be replicated in vivo. Furthermore, the high biological content of this new screening strategy might contribute to improvements in the prediction of the clinical efficacy of compounds in preclinical phases of drug testing and could lead to the identification of further promising targets.

Katherine Whalley

ORIGINAL RESEARCH PAPER Wang, J. K. T. et al.

Cardiac glycosides provide neuroprotection against ischemic stroke: Discovery by a brain slice-based compound screening platform. Proc. Natl Acad. Sci. USA 103, 10461-10466 (2006)

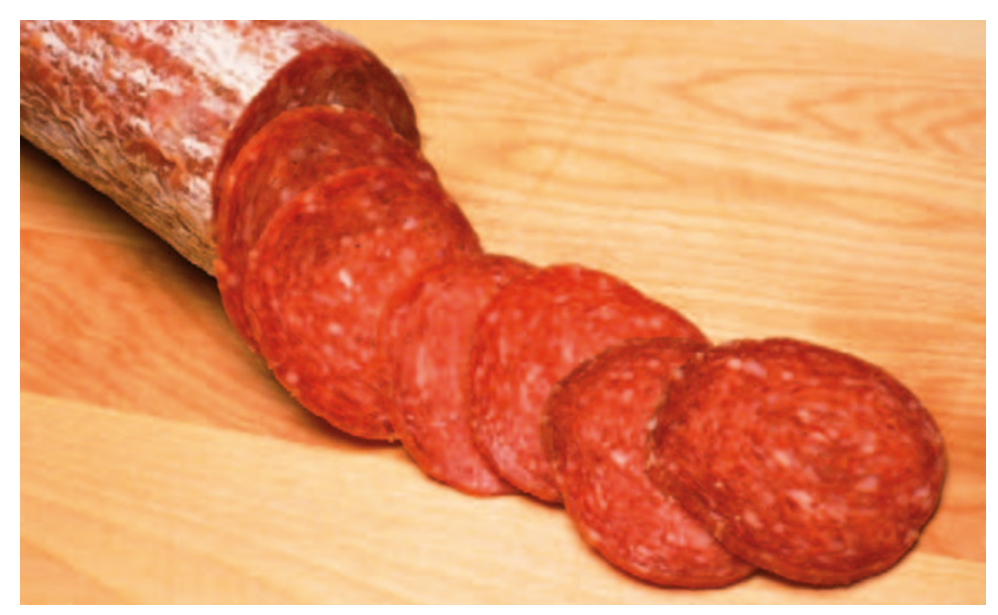

